\title{
Fertilización en rambután (Nephelium lappaceum L.) para mejorar la calidad de frutos
}

\section{Fertilization in rambutan (Nephelium lappaceum L.) for improving the quality of the fruits}

\begin{abstract}
Manuel Reyes-Moreno ${ }^{1}$,
Eduardo García-Villanueva ${ }^{1}$,

Sergio Chávez-Franco ${ }^{1}$ (D)

Alfonso Muratalla-Lúa ${ }^{1}$ (D),

Jesús Reyes-Moreno ${ }^{1}$,

Jesús Mao Estanislao Aguilar-

Luna $^{2} *$ [D

${ }^{1}$ Fruticultura, Colegio de Post-

graduados Campus Montecillo.

Carretera México-Texcoco Km

36.5, CP. 56230, Estado de

México, México.

${ }^{2}$ Ingeniería Agroforestal, Com-

plejo Regional Norte - Sede

Tetela, Benemérita Universidad

Autónoma de Puebla. Av. Univer-

sidad s/n, Tetela de Ocampo, CP.

73640, Puebla, México.
\end{abstract}

*Autor de correspondencia: mao.aguilar@correo.buap.mx

\section{Nota científica}

Recibido: 09 de agosto de 2019 Aceptado: 09 de marzo de 2020

Como citar: Reyes-Moreno M, García-Villanueva E, ChávezFranco S, Muratalla-Lúa A, Reyes-Moreno J, Aguilar-Luna JME (2020) Fertilización en rambután (Nephelium lappaceum L.) para mejorar la calidad de frutos. Ecosistemas y Recursos Agropecuarios 7(1): e2412. DOI: 10.19136/era.a7n1.2412
RESUMEN. El objetivo del estudio fue evaluar dos fertilizantes aplicados a árboles productivos de rambután (Nephelium lappaceum L.) incluyendo la fertilización edáfica convencional, para incrementar la calidad de frutos. Los tratamientos fueron: Biofertilizante más Bayfolan ${ }^{\circledR}\left(T_{1}\right)$, Biofertilizante $\left(T_{2}\right)$, Bayfolan ${ }^{\circledR}\left(T_{3}\right)$, sin fertilizante $\left(T_{4}\right)$. El diseño experimental fue completamente al azar, con cinco repeticiones y un árbol como unidad experimental. Se cuantificó: apariencia del fruto (AP), color del pericarpio, pérdida de peso, firmeza (F), peso fresco (PF), acidez titulable y ${ }^{\circ} \mathrm{Bx}$. La AP mejoró en $28 \%$ en $T_{1}$ y en $T_{3}$; la combinación de los fertilizantes mejoró la calidad de los frutos, hasta con $16 \%{ }^{\circ} \mathrm{Bx}$ y $10 \%$ de PF. En tratamientos restantes los frutos perdieron peso (hasta $35 \%$ ) y $F$ (hasta $41 \%$ ) a los ocho días después de la cosecha. La fertilización foliar más la fertilización convencional, mejoran la calidad postcosecha y vida de anaquel de los frutos de rambután.

Palabras clave: Color de fruto, firmeza, postcosecha, sólidos solubles.

ABSTRACT. The objective was to evaluate the effect of two fertilizer applied to productive rambutan trees (Nephelium lappaceum L.) including conventional edaphic fertilization, for improving the quality of the fruits. The treatments were: Biofertilizer more Bayfolan ${ }^{\circledR}\left(T_{1}\right)$, Biofertilizer $\left(T_{2}\right)$, Bayfolan ${ }^{\circledR}\left(T_{3}\right)$, without fertilizer $\left(T_{4}\right)$. The experimental design was completely randomized, with five repetitions and a tree as experimental unit. It was measured: appearance of the fruit (AF), color of the pericarp, loss of weight, firmness $(\mathrm{F})$, fresh weight $(\mathrm{FW})$, titratable acidity and ${ }^{\circ} \mathrm{Bx}$. The AP improved by $28 \%$ in $T_{1}$ and in $T_{3}$; the combination of fertilizers improved the quality of the fruit at harvest, up to $16 \%{ }^{\circ} \mathrm{Bx}$ and $10 \%$ in $\mathrm{FW}$. The fruits of the remaining treatments lost weight (up to $35 \%$ ) and $F$ (up to $41 \%$ ) to the eight days after the harvest. Conventional and foliar fertilization improve postharvest quality and shelf life of rambutan fruits.

Key words: Fruit color, firmness, post-harvest, soluble solids. 


\section{INTRODUCCIÓN}

El rambután (Nephelium lappaceum L.) es un frutal de la familia Sapindaceae, originario del sureste asiático e introducido a México a finales de los setentas (Pérez y Jürgen 2004, Avendaño-Arrazate et al. 2011). Actualmente se producen $1120000 \mathrm{t}$ en el mundo, de las cuales México aporta 9253.5 t en 883.5 ha, de las que Chiapas tiene el $94.4 \%$, en la región del Soconusco (SIAP 2016). El fruto se comercializa con estándares mundiales de calidad como el color rojo uniforme, calibre mayor o igual a $30 \mathrm{~g}$, sin deformaciones ni pudriciones causadas por plagas o enfermedades; con contenido de sólidos solubles totales de al menos 18\% (CODEX 2005).

Es un fruto no climatérico, que no continúa madurando una vez retirado del árbol, en consecuencia debe ser cosechado cuando ha llegado a un tamaño, calidad y apariencia visual adecuada para su comercialización y consumo (Avendaño-Arrazate et al. 2018). Los frutos se cortan en madurez de cosecha, con base en el color del pericarpio y espiternos según la variedad (Pérez y Jurgen 2004). En las variedades rojas no necesariamente existe una relación proporcional entre el óptimo de sólidos solubles totales y la mayor intensidad de color, ya que a medida que el fruto madura en el árbol, hay un incremento en la cantidad de sólidos solubles con disminución de la acidez titulable (Caballero-Pérez et al. 2011, Famiani et al. 2015). Por lo que el fruto cosechado en estado inmaduro (pericarpio y espiternos verdes) es ácido y sin dulzura adecuada, mientras que en cosecha tardía el fruto puede ser muy suave por la pérdida de firmeza, con el arilo más seco, y la formación de una cámara de aire entre el arilo y el pericarpio (Osorio-Espinoza et al. 2019). Al respecto, Vargas-Calvo (2003) reportan que el mercado de exportación exige frutos de la más alta calidad, con atributos de tamaño adecuado, color rojo intenso uniforme, forma redonda u ovoide, firmeza, aroma y sabor. La pérdida de la apariencia roja del pericarpio de los frutos después de la cosecha, es causada por la deshidratación de los espiternos. Éstos tienen de 15 a 20 haces vasculares con floema y xilema basal, y gran cantidad de es- tomas que frecuentemente están abiertos, por lo que este fenómeno se relaciona con la pérdida de agua en el fruto (Landrigan et al. 1994). Mientras que la deshidratación se asocia con la desecación y el agrietado del pericarpio, lo que causa degradación celular e induce la acción de enzimas oxidantes sobre las antocianinas (Lichter et al. 2000).

Con respecto al consumidor, este compra los frutos por su apariencia visual, siendo la coloración y el tamaño, los más importantes; aunque no existe limitante legal para la comercialización internacional, los compradores se guían por los estándares establecidos en la Norma para el Rambután, con tres categorías: calidad extra (frutos sin defectos), calidad I (defectos que no excedan el $5 \%$ de la superficie del fruto) y calidad II (defectos que no excedan el 10\% de la superficie del fruto) (CODEX 2005). Es por ello, que el arilo (porción comestible) debe constituir una alta relación en peso del fruto y se debe separar fácilmente de las semillas; mientras que, el color externo del pericarpio debe ser rojo uniforme, aromático y de textura firme (Landrigan et al. 1994, AvendañoArrazate et al. 2011).

El manejo nutrimental del rambután es fundamental durante los dos primeros años de establecimiento del huerto. Por lo cual, se debe ajustar la fertilización en función de los índices de extracción, el rendimiento, nutrimentos del suelo y la materia orgánica; de lo contrario se provoca la formación de árboles poco vigorosos y frutos con baja calidad (Avendaño-Arrazate et al. 2018). En México, de manera convencional solo se aplica fertilización edáfica; en este sentido, Galindo y Loquias (2019) recomiendan, para árboles de ocho años de edad, aplicar $30 \mathrm{~kg}$ de abono (estiércol de animal), $2 \mathrm{~kg}$ de 14-14-14, 1 kg de 45-00-00, 1 kg de 00-00-60, por árbol, repartidos a lo largo del año. Pero Prasittikhet et al. (1996) sugieren aplicaciones foliares con 10-1020 , en etapa de fructificación, para incrementar ${ }^{\circ} \mathrm{Bx}$, grosor en el arilo y tamaño de fruto.

El desbalance nutrimental, disminuye el rendimiento y la calidad de los frutos (Patil et al. 2016), ya que en la etapa de floración y desarrollo de frutos, el árbol utiliza grandes cantidades de carbohidratos (Garcia y Martins 2006); mientras que 
posterior a la cosecha y después de una alta producción, se puede registrar un déficit nutrimental que provoca alternancia en el árbol (Osuna et al. 2008). Por lo anterior, el objetivo del estudio fue evaluar dos fertilizantes aplicados a árboles productivos de rambután (Nephelium lappaceum L.) incluyendo la fertilización edáfica convencional, para incrementar la calidad de frutos.

\section{MATERIALES Y MÉTODOS}

La investigación se realizó de julio 2016 a junio de 2017, en una huerta comercial de rambután 'See-Chompoo' de cinco años de edad, ubicada en Frontera Hidalgo, Chiapas, México (14 ${ }^{\circ} 44^{\prime} 12^{\prime \prime} \mathrm{LN}$, $92^{\circ} 10^{\prime} 33^{\prime \prime}$ LO y $46 \mathrm{msnm}$ ). El clima es cálido subhúmedo, con régimen de lluvias en verano (García 2004). El suelo es profundo del tipo nitosol y planosol con textura franco arenosa (INAFED 2016); sus principales características según la NOM-021-RECNAT2000 (SEMARNAT 2002) son: 5.75 de $\mathrm{pH}, 4.92 \%$ de MO, $37.1 \mathrm{cmol} \mathrm{kg}^{-1}$ en CIC, $1.15 \mathrm{~g} \mathrm{~cm}^{-3}$ en DAP, $0.014 \mathrm{~g} \mathrm{~kg}^{-1}$ de N, $33.3 \mathrm{mg} \mathrm{kg}^{-1}$ de $\mathrm{P}$ y $214 \mathrm{mg} \mathrm{kg}^{-1}$ de K.

El diseño de plantación es de $7 \times 6 \mathrm{~m}$ entre hileras y árboles, respectivamente; con un total 238 árboles ha ${ }^{-1}$, con un sistema de riego por microaspersión, manteniendo la parcela a capacidad de campo durante el año. Todos los árboles se fertilizaron con $300 \mathrm{~g}$ de 17-17-17 (excepto el $\mathrm{T}_{4}$ ) aplicado en el suelo. Los tratamientos aplicados a las unidades experimentales fueron: $\mathrm{T}_{1}$ : Biofertilizante más Bayfolan $\mathbb{R}$ ( $3 \mathrm{~L}$ al $15 \%$ más $30 \mathrm{~mL}$ al $0.3 \%$, respectivamente); $\mathrm{T}_{2}$ : Biofertilizante ( $\mathrm{L}$ al $15 \%$ ); $\mathrm{T}_{3}$ : Bayfolan ${ }^{\circledR}(30 \mathrm{~mL}$ al $0.3 \%) ; \mathrm{T}_{4}$ : testigo (sin fertilizantes).

El biofertilizante se preparó siguiendo las recomendaciones para la elaboración, uso y manejo de abonos orgánicos (MAGAP 2014); diluyendo en $50 \mathrm{~L}$ de agua, $5 \mathrm{~kg}$ de estiércol bovino, $2 \mathrm{~kg}$ de residuos foliares (hojas de rambután), $5 \mathrm{~L}$ de melaza, $1 \mathrm{~kg}$ de ceniza (de árboles de la región), $500 \mathrm{~g}$ urea y $50 \mathrm{~g}$ de levadura (Tabla 1). Esta mezcla se dejó fermentar por 30 días en condiciones anaeróbicas. Cada dos meses (de agosto a febrero), primero se aplicó riego por microaspersión (8:00 h); y por la tarde (17:00 h) se aplicó, debajo de la copa del árbol y sobre el suelo húmedo, $5 \mathrm{~L}$ del biofertilizante diluido al $15 \%$ con una bomba manual de $15 \mathrm{~L}$ sin boquilla. El otro producto utilizado fue Bayfolan ${ }^{\circledR}$ (Bayer, Alemania), durante el mismo periodo en concentración de $0.3 \%$, usando el adherente comercial Bond ${ }^{\circledR}$. Las aplicaciones foliares se realizaron con una bomba manual de $15 \mathrm{~L}$ y boquilla tipo abanico, iniciando el 15 de agosto de 2017; cuatro aplicaciones, desde tres meses antes de la inducción floral y finalizando un mes antes de la cosecha.

Los frutos se cosecharon en madurez comercial, cuando el $90 \%$ del pericarpio tenía un color rojo brillante. Posteriormente se trasladaron en refrigeración al Laboratorio de Fisiología de Postcosecha del Colegio de Postgraduados, Campus Montecillo; donde se uniformizaron y clasificaron por su tamaño, color y grado de madurez. Una vez seleccionados se colocaron en cestos de plástico a temperatura ambiente $\left(26 \pm 2{ }^{\circ} \mathrm{C}\right.$ y $46 \%$ de humedad relativa). Para cada variable de respuesta se evaluaron 50 frutos por tratamiento, en esos frutos se realizaron mediciones cada dos días, durante ocho días (tiempo de anaquel).

Las variables de respuesta evaluadas fueron: Apariencia del fruto: se determinó fotográficamente (cámara Nikon D3400) con base en la apariencia externa del fruto, siendo los frutos dañados aquellos con áreas color café en el pericarpio o espiternos necróticos; utilizando la escala propuesta por Kondo et al. (2002), la cual utiliza como referencia el porcentaje de daño de fruto. Color del pericarpio: se cuantificó con las cartas de color RHS (The Royal Horticultural Society) y los datos se expresaron en valores de Luminosidad (L), Chroma y Hue (McGuire 1992). Pérdida de peso (PP): se consideró como la diferencia del peso inicial $(\mathrm{Pi})$ y el peso final $(\mathrm{Pf})$, hasta los ocho días después de la cosecha; por lo cual, cada dos días los frutos fueron pesados en una balanza electrónica (A\&D, modelo EY-2200) aplicando la fórmula: $\mathrm{PP}=(\mathrm{Pi}-\mathrm{Pf} / \mathrm{Pi}) \times 100$. Acidez titulable: se determinó mediante la metodología propuesta por la AOAC (1998), en la cual se tomaron $5 \mathrm{~g}$ de arilo de cada fruto, se licuó en $50 \mathrm{~mL}$ de agua destilada 


\begin{tabular}{|c|c|c|}
\hline Determinación & Método & Resultado \\
\hline $\mathrm{pH}$ & NMX-FF-109-SCFI-2007 & 6.25 \\
\hline CE ds $\mathrm{m}^{-1}$ & NMX-FF-109-SCFI-2007 & 40.8 \\
\hline $\mathrm{N}\left(\mathrm{g} \mathrm{kg}^{-1}\right)$ & Dumas & 0.40 \\
\hline $\mathrm{P} 2 \mathrm{O} 5\left(\mathrm{~g} \mathrm{~kg}^{-1}\right)$ & Digestión en microondas / ICP & 0.40 \\
\hline $\mathrm{K}\left(\mathrm{g} \mathrm{kg}^{-1}\right)$ & Digestión en microondas / ICP & 5.69 \\
\hline $\mathrm{Ca}\left(\mathrm{g} \mathrm{kg}^{-1}\right)$ & Digestión en microondas / ICP & 1.96 \\
\hline $\operatorname{Mg}\left(\mathrm{g} \mathrm{kg}^{-1}\right)$ & Digestión en microondas / ICP & 0.91 \\
\hline $\mathrm{Na}\left(\mathrm{g} \mathrm{kg}^{-1}\right)$ & Digestión en microondas / ICP & 0.25 \\
\hline $\mathrm{S}\left(\mathrm{g} \mathrm{kg}^{-1}\right)$ & Digestión en microondas / Turbidiométrica & 0.57 \\
\hline $\mathrm{Fe}\left(\mathrm{g} \mathrm{kg}^{-1}\right)$ & Digestión en microondas / ICP & 712 \\
\hline $\mathrm{Cu}\left(\mathrm{g} \mathrm{kg}^{-1}\right)$ & Digestión en microondas / ICP & 13.8 \\
\hline $\operatorname{Mn}\left(\mathrm{g} \mathrm{kg}^{-1}\right)$ & Digestión en microondas / ICP & 69.7 \\
\hline $\mathrm{Zn}\left(\mathrm{g} \mathrm{kg}^{-1}\right)$ & Digestión en microondas / ICP & 42.4 \\
\hline $\mathrm{B}\left(\mathrm{g} \mathrm{kg}^{-1}\right)$ & Digestión en microondas / ICP & 23.8 \\
\hline $\mathrm{MO}(\%)$ & Calcinación & 3.72 \\
\hline Cenizas (\%) & Calcinación & 1.81 \\
\hline C org $(\%)$ & Calcinación & 2.16 \\
\hline Relacion $\mathrm{C} / \mathrm{N}$ & Base seca & 5.44 \\
\hline
\end{tabular}

y se tomó una alícuota de $10 \mathrm{~mL}$ de la solución, a la cual se le agregaron tres gotas de fenolftaleína como indicador para posteriormente ser titulada con $\mathrm{NaOH}(0.01 \mathrm{~N})$ hasta obtener el vire color rosado, el gasto de la solución neutralizante fue transformado como porcentaje de ácido cítrico (AC) de acuerdo a la siguiente ecuación: \% de ácido cítrico $=\mathrm{G}(\mathrm{N})$ (0.064) (100) / A, donde: $\mathrm{G}=\mathrm{mL}$ de $\mathrm{NaOH}$ gastados en la titulación, $\mathrm{N}=$ normalidad del $\mathrm{NaOH}, 0.064=$ miliequivalentes del ácido cítrico y $\mathrm{A}=$ alícuota $0 \mathrm{~mL}$ de jugo tomado para la evaluación. Sólidos solubles totales (SST, ${ }^{\circ} \mathrm{Bx}$ ): se determinaron de acuerdo a la metodología AOAC (1998) utilizando un refractómetro digital modelo PR-100 (escala 0-32); los resultados se expresaron como \% de SST. Firmeza: se determinó por penetrometría utilizando un texturómetro Universal, Force-Five Modelo FDV-30, adaptado con un puntal cónico de $5 \mathrm{~mm}$; esta variable fue evaluada cada dos días durante la vida de anaquel de los frutos y el valor fue reportado en newtons $(\mathrm{Nw})$; y peso fresco: se registró de manera individual en cada uno de los 50 frutos por tratamiento, al día siguiente después de la cosecha, utilizando una balanza digital (Ohaus Modelo AP210).

A los datos que no cumplieron el supuesto de normalidad, se les aplicó la prueba de Kruskal Wallis. Mientras que los datos que cumplieron el supuesto de normalidad y de homogeneidad, se les aplicó un análisis de varianza excepto para el color del pericarpio (oscurecimiento). Cuando se detectaron diferencias significativas entre tratamientos se realizó una comparación múltiple de medias con la prueba de Tukey $(\alpha \leq 0.05)$. La asignación de tratamientos a las unidades experimentales se realizó con el programa estadístico $\mathrm{R}$, mientras que los análisis se realizaron con el software Minitab $18^{\circledR}$.

\section{RESULTADOS Y DISCUSIÓN}

La apariencia del fruto, es una de las variables de calidad más importantes en rambután después de su cosecha; según Srilaong et al. (2002), el oscurecimiento del pericarpio del fruto depende, de la cantidad de agua que pierde por sus protuberancias epidérmicas o espiternos. Se tiene un mayor daño por oscurecimiento de los espiternos conforme aumentan los días a la cosecha, tanto en el testigo $\left(\mathrm{T}_{4}\right)$ como en los frutos producidos en los árboles donde solo se aplicó el Biofertilizante $\left(T_{2}\right)$, el daño fue del $50 \%$, no así para los tratamientos donde solo se aplicó Bayfolan ${ }^{\circledR}\left(T_{3}\right)$ o en combinación con el Biofertilizante $\left(T_{1}\right)$; con diferencias estadísticas significativas $(\alpha \leq 0.05)$ a partir del tercer día, después de la cosecha de fruto (Tabla 2).

En el séptimo día los frutos de mejor apariencia, menor oscurecimiento del pericarpio y espiter- 
Tabla 2. Superficie dañada y pérdida de peso, en frutos de rambután.

\begin{tabular}{|c|c|c|c|c|c|c|c|c|}
\hline \multirow[t]{3}{*}{ Tratamientos } & \multicolumn{4}{|c|}{ SDF (\%) } & \multicolumn{4}{|c|}{ PP (\%) } \\
\hline & \multicolumn{8}{|c|}{ Tiempo después de la cosecha (días) } \\
\hline & 1 & 3 & 5 & 7 & 2 & 4 & 6 & 8 \\
\hline $\mathrm{T}_{1}$ : Biofertilizante + Bayfolan $\left.{ }^{\circledR}\right)$ & 0 & $09^{b B}$ & $22^{b A}$ & $24^{c A}$ & $2.6^{a D}$ & $7.7^{b C}$ & $16.3^{b B}$ & $23.5^{c A}$ \\
\hline $\mathrm{T}_{2}:$ Biofertilizante & 0 & $27^{a B}$ & $46^{a A}$ & $50^{a A}$ & $3.6^{a D}$ & $9.8^{a b C}$ & $21.5^{a B}$ & $30.2^{b A}$ \\
\hline $\mathrm{T}_{3}:$ Bayfolan ${ }^{\mathrm{B}}$ & 0 & $12^{a b C}$ & $24^{b B}$ & $37^{b A}$ & $3.2^{a D}$ & $9.0^{a b C}$ & $21.8^{a B}$ & $27.8^{b A}$ \\
\hline $\mathrm{T}_{4}$ : Testigo & 0 & $26^{a B}$ & $48^{a A}$ & $50^{a A}$ & $4.6^{a D}$ & $11.9^{a C}$ & $24.8^{a B}$ & $35.0^{a A}$ \\
\hline
\end{tabular}

SDF: superficie dañada en frutos. PP: pérdida de peso en frutos almacenados a temperatura ambiente.
Medias con literales minúsculas diferentes presentan diferencias entre tratamientos. Medias con literales mayúsculas diferentes presentan diferencias dentro del tratamiento (Tukey, $\alpha \leq 0.05$ ).

nos más firmes, fueron los del $T_{1}$; mientras que los frutos que presentaron una apariencia comercial no aceptable según el CODEX (2005), fueron los del $\mathrm{T}_{2}$, $\mathrm{T}_{3}$ y $\mathrm{T}_{4}$ (Figura 1). La vida de anaquel del fruto de rambután está limitada por la susceptibilidad del pericarpio a la desecación y oscurecimiento (Patil et al. 2016). Al respecto, Landrigan et al. (1996) mencionan que cuando el fruto muestra pérdidas de peso mayores al $20 \%$, la oxidación se hace evidente en los espiternos de donde se extiende hacia el pericarpio; ya en etapas avanzadas de deshidratación la apariencia cambia de forma negativa. Al respecto, Hassan et al. (2010) indican que al asperjar compuestos orgánicos y micronutrimentos quelatados se expresa un efecto favorable en el amarre, el peso de fruto y por tanto en el rendimiento; debido a que los metabolitos o compuestos bacterianos presentes en los biofertilizantes, hacen más eficiente la absorción nutrimental (Radzki et al. 2013).

La fertilización foliar es un complemento importante en la nutrición del rambután, debido al aporte de nutrimentos en etapas críticas previas a la cosecha de frutos (Mandal et al. 2012); ya que un incremento en la relación $\mathrm{K} / \mathrm{N}$ prolonga la vida postcosecha inhibiendo el oscurecimiento del pericarpio, lo que disminuye la permeabilidad de la membrana del pericarpio, inhibiendo la actividad de la polifenol oxidasa y de la peroxidasa (Yang et al. 2015). En Tabla 2, se observa que la pérdida de peso en frutos de rambután, se debe principalmente a la pérdida de agua o deshidratación; ya que el peso disminuye conforme pasa el tiempo después de la cosecha. A partir del cuarto y hasta los ocho días, el tratamiento $T_{1}$ fue el que menos peso perdió (11.5\%) comparado con el $\mathrm{T}_{4}$ que tuvo mayor pérdida de peso del $35 \%$. A los ocho días después de la cosecha los frutos a los que se les asignó algún tratamiento, presentaron la mayor deshidratación siendo esta superior hasta un 32.9\% en comparación con el mejor tratamiento $\left(T_{1}\right)$. Una respuesta similar encontraron Landrigan et al. (1996), quienes reportan pérdidas de peso entre el 23 y $35 \%$ al octavo día, sugiriendo que esta se relaciona con la pérdida de agua durante la transpiración de los frutos; debido a la presencia de espiternos en el pericarpio, los cuales presentan entre 50 y 70 estomas por $\mathrm{mm}^{2}$; respuesta similar fue reportada por AvendañoArrazate et al. (2011) quienes encontraron una gran cantidad de estomas que permanecen abiertos y permiten la pérdida de agua sin control de los espiternos.

Los frutos de rambután contienen diferentes tipos de ácidos en el arilo, predominando el ácido cítrico y en menor cantidad el ácido succínico (Sivakumar et al. 2000, Avendaño-Arrazate et al. 2018). En la Tabla 3, se muestra el efecto de los tratamientos en la concentración de ácidos orgánicos, expresada en porcentaje de acidez titulable, no habiendo diferencia estadística significativa ( $\alpha \leq$ 0.05 ) a los 1,3 y 5 días después de la cosecha; pero si hubo cambios a los siete días, ya que la concentración de ácidos orgánicos se incrementó un $0.08 \%$ en $\mathrm{T}_{2}$ y $\mathrm{T}_{4}$. Al respecto, Tongtao et al. (2015) afirman que el comportamiento del ácido cítrico en la pulpa del rambután (arilo) no es consistente posterior a la cosecha, ya que los niveles suben o bajan con el almacenamiento de los frutos; lo cual está asociado con la disminución del ácido málico, que representa el $80 \%$ del total de los ácidos, y el incremento de otros ácidos: cítrico, succínico, levulínico, glutárico, malónico y láctico (Famiani et al. 2015). Por lo cual, la fertilización es importante ya que puede evitar el 
día 5
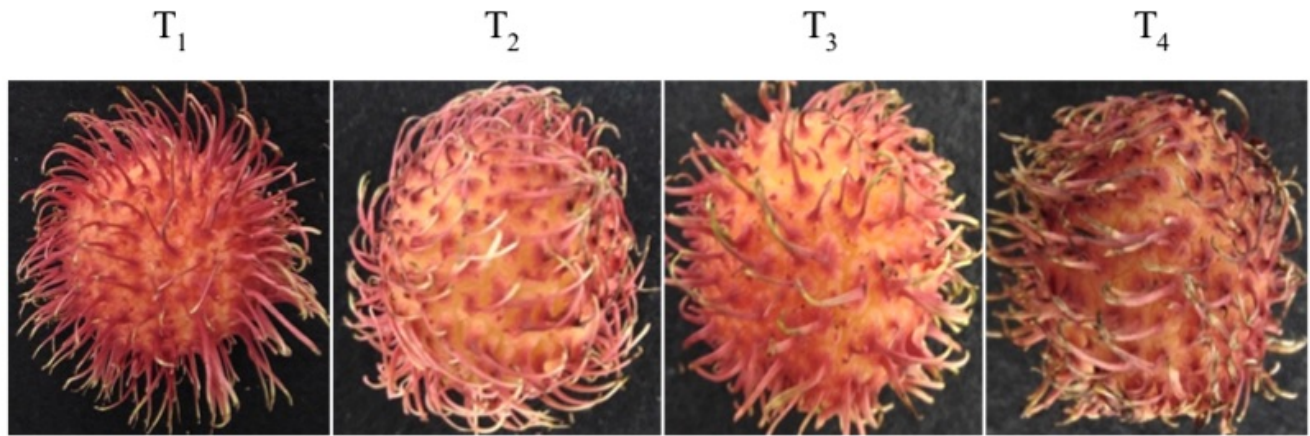

día 7
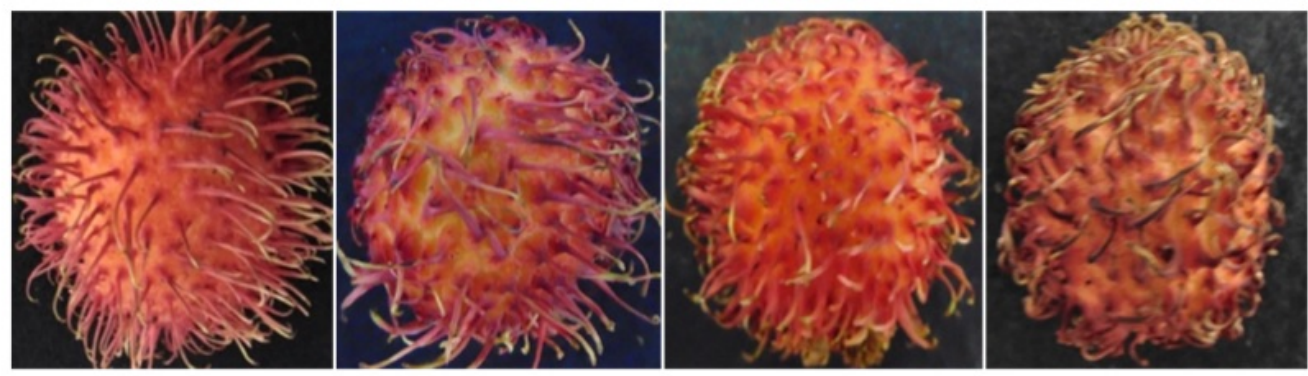

Figura 1. Oscurecimiento del pericarpio en frutos de rambután, a los cinco y siete días después de su cosecha, almacenados a temperatura ambiente $\left(26 \pm 2{ }^{\circ} \mathrm{C}\right.$ y $46 \%$ de humedad relativa).

estrés del árbol, debido a la estrecha relación que hay entre el metabolismo de los ácidos orgánicos y el estrés. Al respecto, Walker y Famiani (2018) concluyen que los ácidos orgánicos son importantes en la absorción y asimilación de $\mathrm{N}$ en forma de $\mathrm{NO}_{3}$, siendo determinantes en el intercambio activo de la raíz y el funcionamiento de la nitrato y nitrito reductasa para la reducción de $\mathrm{NH}_{4}$ asimilable.

Desde el primer día se observaron cambios en la concentración de ${ }^{\circ} \mathrm{Bx}$, con diferencia estadística entre tratamientos $(\alpha \leq 0.05)$, y valores iniciales de 16.9 a 20.1, que se modificaron en la medida que transcurrió el tiempo. Hasta los ocho días después de la cosecha, la mayor concentración acumulada fue de 19.8 , siendo $T_{1}$ (Biofertilizante + Bayfolan ${ }^{\circledR}$ ) y $T_{3}$ (Bayfolan $(B)$ ) los que mejor respuesta ofrecieron, incrementando los ${ }^{\circ} \mathrm{Bx}$ hasta en un $9.5 \%$ con respecto de los frutos provenientes de árboles no fertilizados (Tabla 3). Al respecto, Vargas-Calvo (2003) reportan valores de 18.3 a $18.6{ }^{\circ} \mathrm{Bx}$ en frutos de cinco cultivares de rambután. Por otro lado, Hernández-Arenas et al. (2012) concluyen que los sólidos solubles totales disminuyen de 19.3 hasta $18^{\circ} \mathrm{Bx}$ a los 14 días de almacenamiento, sobre lo mismo Nampan et al. (2006) recomiendan que los frutos sean tratados con hidroenfriamiento para hacer más lento el metabolismo y prolongar la vida postcosecha.

La firmeza también es un indicador del grado de deshidratación del pericarpio, con el consecuente oscurecimiento y pérdida de calidad visual en los frutos (Hernández-Arenas et al. 2012). En la Tabla 4 , se observa que a mayor tiempo de cosecha, la firmeza del pericarpio del fruto disminuye; siendo los frutos provenientes de árboles no tratados los que presentan menor firmeza desde la cosecha, con valores de 2.8 y $2.3 \mathrm{Nw}$ entre la primera y la última fecha de evaluación; es decir, estos perdieron el $18 \%$ de la firmeza $\left(T_{4}\right)$. Pero cuando se combina una fuente de fertilización orgánica y otra química vía foliar, aumenta la firmeza de los frutos hasta en un $29 \%$ en la cosecha, manteniendo la tendencia desde la cosecha hasta que termina su vida de anaquel, aunque ésta puede prolongarse hasta 14 días en atmósferas modificadas (Hernández-Arenas et al. 2012) y con hidroenfriamiento (Nampan et al. 2006).

Con respecto del peso fresco de los frutos, fue el tratamiento $T_{1}$ (Biofertilizante más Bayfolan ${ }^{\circledR}$ ) el 
Tabla 3. Acidez titulable y porcentaje de sólidos solubles totales, en frutos de rambután.

\begin{tabular}{|c|c|c|c|c|c|c|c|c|}
\hline \multirow[t]{3}{*}{ Tratamientos } & \multicolumn{4}{|c|}{ AT (\%) } & \multicolumn{4}{|c|}{$\mathrm{SST}\left({ }^{\circ} \mathrm{Bx}\right)$} \\
\hline & \multicolumn{8}{|c|}{ Tiempo después de la cosecha (días) } \\
\hline & 1 & 3 & 5 & 7 & 2 & 4 & 6 & 8 \\
\hline $\mathrm{T}_{1}$ : Biofertilizante + Bayfolan ${ }^{(\mathrm{B})}$ & $0.53^{a A}$ & $0.51^{a A}$ & $0.54^{a A}$ & $0.54^{b A}$ & $20.1^{a A}$ & $19.2^{a A}$ & $19.6^{a A}$ & $19.8^{a A}$ \\
\hline $\mathrm{T}_{2}:$ Biofertilizante & $0.50^{a B}$ & $0.52^{a B}$ & $0.56^{a A B}$ & $0.58^{a A}$ & $18.6^{b A}$ & $18.7^{a b A}$ & $19.0^{a b A}$ & $18.4^{b A}$ \\
\hline $\mathrm{T}_{3}$ : Bayfolan ${ }^{\circledR}$ & $0.50^{a A}$ & $0.50^{a A}$ & $0.52^{a A}$ & $0.53^{b A}$ & $18.7^{b A B}$ & $19.5^{a A}$ & $18.2^{b B}$ & $19.6^{a A}$ \\
\hline $\mathrm{T}_{4}$ : Testigo & $0.51^{a B}$ & $0.54^{a A B}$ & $0.55^{a A B}$ & $0.58^{a A}$ & $16.9^{c B}$ & $18.6^{b A}$ & $17.2^{c A B}$ & $17.9^{b A}$ \\
\hline
\end{tabular}

Tabla 4. Firmeza (Nw) en frutos de rambután durante siete días de almacenamiento a temperatura ambiente.

\begin{tabular}{lcccc}
\hline & \multicolumn{4}{c}{ Tiempo después de la cosecha (días) } \\
\cline { 2 - 5 } Tratamientos & 1 & 3 & 5 & 7 \\
\hline $\mathrm{T}_{1}$ : Biofertilizante + Bayfolan ${ }^{\circledR}$ & $4.4^{a A}$ & $4.1^{a A}$ & $4.0^{a A}$ & $3.9^{a A}$ \\
$\mathrm{~T}_{2}$ : Biofertilizante & $3.4^{c A}$ & $3.4^{b A}$ & $3.2^{b A}$ & $3.0^{b A}$ \\
$\mathrm{~T}_{3}$ : Bayfolan ${ }^{\circledR}$ & $4.1^{b A}$ & $3.5^{b B}$ & $3.3^{b B}$ & $3.1^{b B}$ \\
$\mathrm{~T}_{4}$ : Testigo & $2.8^{d A}$ & $2.7^{c A B}$ & $2.5^{c A B}$ & $2.3^{b B}$ \\
\hline
\end{tabular}

Medias con literales minúsculas diferentes presentan diferencias entre tratamientos. Medias con literales mayúsculas diferentes presentan diferencias dentro del tratamiento (Tukey, $\alpha \leq 0.05$ ).

que mostró un valor superior (32.0 g) con respecto de los demás tratamientos, inmediatamente seguidos por los árboles tratados solo con Bayfolan ${ }^{\circledR}$ vía foliar $(30.4 \mathrm{~g})$ y finalmente los árboles tratados solo con el Biofertilizante, siendo el testigo el de menor valor (Tabla 5). Lo anterior, puede atribuirse a una mayor concentración de nutrimentos en los tejidos vegetales. Al respecto, Fallahi et al. (2002) afirman que la fertilización al suelo presenta algunas desventajas, sobre todo en etapas críticas del cultivo como la inducción floral y primeros estadios en el desarrollo de frutos, ya que muchos de los nutrimentos proporcionados se pierden por lixiviación, por lo que la fertilización foliar es una alternativa en términos de oportunidad y eficiencia. Sobre lo mismo Saucedo-Hernández et al. (2005) mencionan que los frutos pre tratados con $\mathrm{Ca}^{2+}$ y posteriormente almacenados, disminuyen su pérdida de peso y mantienen su firmeza. Por lo cual, las aplicaciones foliares con Bayfolan ${ }^{\circledR}$ pueden mejorar las características postcosecha de los frutos de rambután; pues según la hipótesis del efecto ácido en el crecimiento, las auxinas estimulan la actividad de la bomba de protones (H+-ATPasa) localizada en la membrana plasmática. Así, la extracción de protones hacia la pared celular genera una reducción del pH, que a su vez ac- tiva proteínas que rompen enlaces de hidrógeno entre los constituyentes de la pared (Zhang et al. 2019). Los candidatos más probables para este papel inicial son las expansinas, proteínas de pared que favorecen la plasticidad de la célula; otras enzimas hidrolíticas actúan posteriormente y la célula crece como resultado de la presión de turgor generada por la vacuola y por el depósito de nuevos materiales, cuya síntesis y transporte también parecen estar regulados por las auxinas (Martínez-González et al. 2017). De manera similar, Avendaño-Arrazate et al. (2011) concluyen que para lograr un buen desarrollo en los árboles de rambután y obtener rendimientos óptimos, es necesario la elaboración de un programa de fertilización; en función de las demandas para obtener frutos de calidad, que incluya en orden de importancia N, K, Ca, Mg y S (Vargas-Calvo 2003), ya que en la maduración de los frutos con calidad se activa todo un conjunto de rutas bioquímicas que hacen que éste sea atractivo y deseable para los consumidores (Martínez-González et al. 2017) y al incrementar la relación $\mathrm{K} / \mathrm{N}$ se puede prolongar la vida postcosecha (Yang et al. 2015). Por lo tanto, la fertilización edáfica convencional con $300 \mathrm{~g}$ por árbol de 17-17-17, aunada a la fertilización foliar mejoran la calidad y vida postcosecha de los frutos de rambután. 
Tabla 5. Peso fresco de un fruto de rambután (un día después de la cosecha).

\begin{tabular}{lc}
\hline Tratamientos & Peso fresco $(\mathrm{g})$ \\
\hline T1: Biofertilizante + Bayfolan $\AA$ ) & $32.0^{a}$ \\
T2: Biofertilizante & $28.6^{b}$ \\
T3: Bayfolan $\AA$ & $30.4^{a b}$ \\
T4: Testigo & $28.8^{b}$ \\
\hline Medias con distinta letra en la misma columna son \\
estadísticamentre diferentes (Tukey, $\alpha \leq 0.05$ ).
\end{tabular}

\section{LITERATURA CITADA}

AOAC (1998) Official Methods of Analysis. Association of Official Analytical Chemist. 16th edition. AOAC International. Gaithersburg, MD. USA. 1298p.

Avendaño-Arrazate $\mathrm{CH}$, Arévalo-Galarza ML, Sandoval-Esquivez A, Caballero-Pérez JF (2011) El rambután (Nephelium lappaceum) un cultivo con amplio potencial de exportación en el sur de México. Agroproductividad 4: 9-17.

Avendaño-Arrazate CH, Moreno-Pérez EC, Martínez-Damián MT, Cruz-Alvarez O, Vargas-Madríz H (2018) Postharvest quality and behavior of rambutan (Nephelium lappaceum L.) fruits due to the effects of agronomic practices. Revista Chapingo Serie Horticultura 24: 13-26.

Caballero-Pérez JF, Arévalo-Galarza ML, Avendaño-Arrazate CH, Cadena-Iñiguez J, Valdovinos-Ponce G, Aguirre-Medina JF (2011) Cambios físicos y bioquímicos durante el desarrollo y senescencia de frutos de rambután (Nephelium lappaceum L.). Revista Chapingo Serie Horticultura 17: 31-38.

CODEX (2005) Norma del CODEX para el rambután. CODEX STAN 246-2005, México. 4p.

Fallahi E, Khemira H, Righetti TL, Azarenko AN (2002) Influence of foliar application of urea on tree growth, fruit quality, leaf minerals, and distribution of urea-derived nitrogen in apples. Acta Horticulturae 594: 603-610.

Famiani F, Battistelli A, Moscatello S, Cruz-Castillo JG, Walker RP (2015) The organic acids that are accumulated in the flesh of fruits: occurrence, metabolism and factors affecting their contents - a review. Revista Chapingo Serie Horticultura 21: 97-128.

Galindo RA, Loquias MP (2019) Rambutan production guide. In: Low K (ed.) Asian fruits and berries: growing them, eating them, appreciating their lore. Mc Farland \& Company, Inc., Publishers. North Carolina, USA. pp: 203-207.

García E (2004) Modificaciones al sistema de clasificación climática de Köppen. 5a edición. Instituto de Geografía, Universidad Nacional Autónoma de México, México. 93p.

Garcia PE, Martins GAB (2006) Flowering and fruiting of lychee trees in response to girdling of branches. Revista Brasileira de Fruticultura 28: 14-17.

Hassan HSA, Sarrwy SMA, Mostafa EAM (2010) Effect of foliar spraying with liquid organic fertilizer, some micronutrients, and gibberellins on leaf mineral content, fruit set, yield, and fruit quality of "Hollywood" plum trees. Agriculture and Biology Journal of North America 1: 638-643.

Hernández-Arenas M, Nieto-Ángel D, Martínez-Damián MT, Teliz-Ortiz D, Nava-Díaz C, Bautista-Martínez N (2012) Almacenamiento postcosecha de rambután en dos temperaturas y atmósferas modificadas. Interciencia 37: 542-545. 
INAFED (2016) Instituto nacional para el federalismo y el desarrollo municipal. http://www.inafed.gob.mx/work/ enciclopedia/EMM07chiapas/index.html. Fecha de consulta: 22 de noviembre de 2017.

Kondo S, Nimitkeatkai H, Kanlayanarat S (2002) Cell wall metabolism during development of rambutan fruit. Journal of Horticultural Science and Biotechnology 77: 300-304.

Landrigan M, Sarafis V, Morris S, McGlasson W (1994) Structural aspects of rambutan (Nephelium lappaceum) fruits and their relation to postharvest browning. Journal of Horticultural Science 69: 571-579.

Landrigan M, Morris SC, Eamus D, McGlasson WB (1996) Postharvest water relationships and tissue browning of rambutan fruit. Scientia Horticulturae 66: 201-208.

Lichter A, Dvir O, Rot I, Akerman M, Regev R, Wiesblum A, Fallik E, Zauberman G, Fuchs Y (2000) Hot water brushing: an alternative method to SO2 fumigation for color retention of litchi fruits. Postharvest Biology and Technology 18: 235-244.

MAGAP (2014) Elaboración, uso y manejo de abonos orgánicos. Ministerio de Agricultura, Ganadería, Acuacultura y Pesca, Ecuador. 20p.

Mandal G, Dhaliwal HS, Mahajan BV (2012) Effect of pre-harvest application of NAA and potassium nitrate on storage quality of winter guava (Psidium guajava). Indian Journal of Agricultural Sciences 82: 985-989.

Martínez-González ME, Balois-Morales R, Alia-Tejacal I, Cortes-Cruz MA, Palomino-Hermosillo YA, López-Gúzman GG (2017) Postharvest fruits: maturation and biochemical changes. Revista Mexicana de Ciencias Agrícolas 19: 4075-4087.

McGuire RG (1992) Reporting of objective color measurements. HortScience 27: 1254-1255.

Nampan K, Techavuthiporn C, Kanlavanarat S (2006) Hydrocooling improves quality and storage life of 'RongRein' rambutan (Nephelium lappaceum L.) fruit. Acta Horticulturae 712: 763-770.

Osorio-Espinoza H, Leyva-Galan A, Toledo-Toledo E, Marroquín-Agreda FJ, Gabriel-Hernandez M (2019) La producción de rambután (Nephelium lappaceum L.) en Chiapas, México. Oportunidades para una producción agroecológica. Cultivos Tropicales 40: a14-e14.

Osuna ET, Valenzuela RG, Muy RMD, Gardea BAA, Villareal RM (2008) Expresión del sexo y anatomía floral del litchi (Litchi chinensis Sonn.). Revista Fitotecnia Mexicana 31: 51-56.

Patil NN, Mishra NK, Singh CP, Srivastava R, Singh AK (2016) Influence of pre-harvest foliar application of nutrients and growth regulators on fruit quality of litchi (Litchi chinensis Sonn.) cv. Rose Scented. HortFlora Research Spectrum 5: 124-128.

Pérez A, Jurgen HA (2004) Prácticas de cosecha y poscosecha del rambután, en el Soconusco, Chiapas, México. Leisa Revista de Agroecología 20: 24-26.

Prasittikhet J, Sangtong P, Chongpraditnum P (1996) Effect of fertilizer grades on growth, yield and quality of rambutan. In: Proceedings of the international conference on tropical fruits: global commercialization of tropical fruits: Kuala Lumpur, Malaysia. pp: 105-115.

Radzki W, Gutierrez-Mañero FJ, Algar E, Lucas-Garcia JA, Garcia-Villaraco A, Ramos-Solano B (2013) Bacterial siderophores efficiently provide iron to iron-starved tomato plants in hydroponics culture. Antonie van Leeuwenhoek 104: 321-330.

Saucedo-Hernández L, Martínez-Damián MT, Colinas-León MT, Barrientos-Priego AF, Aguilar-Melchor JJ (2005) Aplicaciones foliares de nitrato de calcio en la maduración y daños por frío en aguacate 'Fuerte'. Revista Chapingo Serie Horticultura 11: 149-157. 
SEMARNAT (2002) Norma Oficial Mexicana NOM-021-RECNAT-2000, que establece las especificaciones de fertilidad, salinidad y clasificación de suelos. Estudios, muestreo y análisis. Secretaría de Medio Ambiente y Recursos Naturales http://extwprlegs1.fao.org/docs/pdf/mex50674.pdf. Fecha de consulta: 22 de noviembre de 2017.

SIAP (2016) Servicio de Información Agroalimentaria y Pesquera. http://nube.siap.gob.mx/cierre_agricola. Fecha de consulta: 22 de noviembre de 2017.

Sivakumar D, Wijeratnam SW, Wijesundera RLC, Abeyesekere M (2000) Antagonistic effect of Trichoderma harzianum on postharvest pathogens of rambutan (Nephelium lappaceum). Phytoparasitica 28: 240-247.

Srilaong V, Kanlayanarat S, Tatsumi Y (2002) Changes in commercial quality of 'Rong-Rien' rambutan in modified atmosphere packaging. Food Science and Technology Research 8: 337-341.

Tongtao S, Srilaong V, Kanlayanarat S, Noichinda S, Bodhipadma K, Khumjareon S, Leung DWM (2015) Sugar status at harvest and during postharvest storage of Nephelium lappaceum cv. 'Sri-Chompoo' fruit from different maturity stages. International Food Research Journal 22: 190-195.

Vargas-Calvo A (2003) Descripción morfológica y nutricional del fruto de rambután (Nephelium lappaceum). Agronomía Mesoamericana 14: 201-206.

Walker RP, Famiani F (2018) Organic acids in fruits: metabolism, functions and contents. Horticultural Reviews 45: 371-430. DOI: 10.1002/9781119431077.ch8

Yang B, Li G, Yang S, He Z, Zhou C, Yao L (2015) Effect of application ratio of potassium over nitrogen on litchi fruit yield, quality, and storability. HortScience 50: 916-920.

Zhang S, Lin H, Lin M, Lin Y, Chen Y, Wang H, Lin Y, Shi J (2019) Lasiodiplodia theobromae (Pat.) Griff. \& Maubl. Reduced energy status and ATPase activity and its relation to disease development and pericarp browning of harvested longan fruit. Food Chemistry 275: 239-245. 